\title{
Developing Heterotic Groups for Successful Hybrid Breeding in Perennial Ryegrass
}

\author{
Maximilian Vogt ${ }^{1,2}{ }^{\oplus}$, Steven Yates ${ }^{1}$, Timothy Sykes ${ }^{1} \oplus$, Wilbert Luesink ${ }^{3}$, Michael Koch $^{2}$ and \\ Bruno Studer 1 * $* \mathbb{D}$ \\ 1 Molecular Plant Breeding, Institute of Agricultural Sciences, ETH Zurich, Universitaetstrasse 2, \\ 8092 Zurich, Switzerland; maximilian.vogt@usys.ethz.ch (M.V.); steven.yates@usys.ethz.ch (S.Y.); \\ timothy.sykes@fgcz.ethz.ch (T.S.) \\ 2 Deutsche Saatveredelung AG, Weissenburger Strasse 5, D-59557 Lippstadt, Germany; \\ michael.koch@dsv-saaten.de \\ 3 Norddeutsche Pflanzenzucht Hans-Georg Lembke KG, Hohenlieth-Hof 1, D-24363 Holtsee, Germany; \\ w.luesink@npz.de \\ * Correspondence: bruno.studer@usys.ethz.ch; Tel.: +41-44-632-01-57
}

Received: 12 July 2020; Accepted: 15 September 2020; Published: 17 September 2020

\begin{abstract}
Perennial ryegrass (Lolium perenne L.), an important forage grass species in temperate regions, is genetically improved by population breeding. Although valued for their broad genetic base, the resulting synthetic varieties only partially exploit heterosis. Hybrid breeding offers opportunities to fix beneficial heterotic patterns more effectively and, hence, to increase the yield potential. A suspected bottleneck in the production of perennial ryegrass hybrids is the genetic intermixture of existing germplasm, impeding the definition of heterotic groups. In this study, selected parental populations of a diploid and tetraploid cytoplasmic male sterility (CMS)-based hybrid breeding program were characterized using genotyping-by-sequencing (GBS). Hybrid populations, derived from 26 parental combinations of the tetraploid breeding program, were tested for yield performance and compared to synthetic varieties at five sites over four growing seasons. The hybrids significantly outperformed the synthetics by $4.15 \%$ on average for total dry matter yield. Additionally, GBS revealed the existence of sub-populations within the tetraploid CMS germplasm. This sub-population structure represents the untapped potential that could be exploited for heterosis to further increase biomass yields. Here, we show that CMS hybrids generate substantial yield gains in perennial ryegrass and provide a method to further improve hybrid breeding, using GBS to select for heterotic groups.
\end{abstract}

Keywords: genotyping-by-sequencing (GBS); grassland; heterosis; heterotic groups; hybrid breeding; perennial ryegrass (Lolium perenne $\mathrm{L}$.)

\section{Introduction}

In temperate regions, perennial ryegrass (Lolium perenne L.) is the predominantly grown forage grass, supplying a major fraction of the roughage for beef and milk production [1]. It is valued for its high fodder quality and persistency but lacks reliable tolerance to cold, heat, and drought stress $[1,2]$. The development of hardy tetraploid varieties has mitigated some of these problems [2]. However, further improvement is needed as perennial ryegrass yields have recently suffered from weather extremes, damaging pastures across central Europe [3,4].

For allogamous forage grasses, the most widely used breeding strategy to produce cultivars is synthetic breeding $[5,6]$. Besides, in perennial ryegrass, this breeding strategy has proven successful for cultivar development and to meet the requirements for distinctiveness, uniformity, and stability (DUS) criteria [5]. At the beginning of each breeding cycle, 8-16 plants are selected for a population cross. 
Ideally, the newly formed synthetic variety shows uniform phenotypic appearance and behavior but is genetically intermixed [6-8]. To broaden the genetic diversity within breeding programs, plants are often shared between breeding companies [9]. Occasionally, sharing is the result of collaborations, but mostly, plants are selected from available cultivars by making use of breeders' privilege $[9,10]$. This practice leads to genetically-related plant material within and between breeding programs. Consequently, the origins and pedigrees of perennial ryegrass cultivars are vague or untraceable, especially when competitors' material is used [11]. Besides, the ancestry of new genetic material is rarely considered because the selection of new genotypes is mainly based on the plant's phenotype and its ability to fit into the population crosses of the synthetic breeding program. While the resulting cultivars are valued for their broad genetic base, only low genetic gains for dry matter yield of $0.25-0.6 \%$ per year have been realized through this method [12].

A transition from synthetic to hybrid breeding is considered as a way to improve the yield potential of perennial ryegrass [5,12]. The main goal of classical hybrid breeding is exploiting heterosis, whereby the crossing of two homozygous plants generates a heterozygous F1 hybrid, which outperforms the parental means [13]. One requirement for the cost-effective production of hybrid seeds is a stable system to control pollination [14]. Existing pollination control strategies in perennial ryegrass include cytoplasmic male sterility (CMS) or self-incompatibility (SI) $[8,12,15]$. In addition to pollination control, hybrid vigor is increased when heterosis is maximized by combining divergent germplasm [16,17]. However, well-defined and divergent germplasm is not established yet, limiting effective hybrid breeding in perennial ryegrass $[5,11,18]$. Classically, individuals or populations are defined as a heterotic group when they show similar hybrid performance when crossed to genetically different groups $[16,17]$. The degree of heterosis exploited through the combination of heterotic groups reflects the heterotic pattern within the structured germplasm. Genetically-structured germplasm enhances the efficiency of hybrid breeding programs, as it enables breeders to predict successful crosses and, thus, reduces the number of test-crosses required to optimize heterosis [12,17].

Achievements of hybrid breeding in maize (Zea mays L.), canola (Brassica napus L.), and rye (Secale cereale L.) demonstrate its superior performance over other breeding strategies [19-22]. Rye serves as a model for hybrid breeding in perennial ryegrass. Firstly, like ryegrasses, rye is an outcrossing species [22,23]. Secondly, CMS systems have been exploited in the rye [24]. But unlike perennial ryegrass, rye populations have been separated into heterotic groups [21]. The continuous development of these groups has allowed breeders to diverge female and male heterotic groups. These developments have resulted in hybrids with up to $30 \%$ increased yield over synthetic varieties [21,25]. In perennial ryegrass, clear identification of heterotic groups within germplasm of different origins has proven difficult, even with molecular tools [26]. If the same benefits of hybrid breeding seen in rye are to be realized in perennial ryegrass, the identification and demarcation of heterotic groups are essential $[11,26]$.

The objectives of this study were (1) to develop a method for high-throughput genotyping of both diploid and tetraploid perennial ryegrass populations, (2) to characterize the genetic structure of selected parental populations currently used for hybrid breeding, (3) to provide an applicable method to further separate parental populations for the development of heterotic groups, and (4) to investigate the performance of CMS-based hybrids in comparison to synthetic varieties of perennial ryegrass.

\section{Materials and Methods}

\subsection{Plant Material}

The plant material used in this study belongs to the joint perennial ryegrass hybrid breeding program of Deutsche Saatveredelung AG (DSV) and Norddeutsche Pflanzenzucht Hans-Georg Lembke KG (NPZ). The four parental populations selected for genotyping-by-sequencing (GBS) analysis comprised one diploid CMS population, one diploid pollinator population, one tetraploid CMS 
population, and one tetraploid pollinator population. For the analysis of the genetic structure, 60 individuals of each of the four populations were randomly selected.

The yield trials consisted of 26 tetraploid hybrid populations, derived from 26 parental combinations of the tetraploid CMS hybrid breeding program. The 26 tetraploid parental populations (nine CMS and 17 pollinator populations, among them, the two used for genetic structure analysis) were crossed following a company-specific matrix scheme to produce F1-hybrid seeds. F1-hybrids were harvested from the CMS populations. Nine tetraploid synthetic varieties were used as standards within the yield trials. The populations, as well as yield trial information, were anonymized according to the common practice of the owner.

\subsection{Genotyping-by-Sequencing}

The tissue of young leaves was harvested with three pieces of $1 \mathrm{~cm}$ for each plant. DNA was extracted using a 96-well plate KingFisher Flex Purification System (Thermo Fisher Scientific, Waltham, MA, USA) with Mag-Bind ${ }^{\circledR}$ Plant DNA DS 96 Kit (Omega Bio-tek, Inc., Norcross, GA, USA). The quality of isolated DNA was assessed on a 1\% agarose gel and quantified with a NanoDrop 8000 spectrophotometer (Thermo Fisher Scientific, Waltham, MA, USA).

The GBS libraries were prepared using restriction enzyme-digested (PstI, Thermo Fisher Scientific, Waltham, MA, USA) genomic DNA, multiplexed by 192 unique 5-10 bp long barcodes. These barcodes were designed with the Deena Bioinformatics online GBS Barcode Generator (http://www.deenabio. $\mathrm{com} / \mathrm{nl} /$ services/gbs-adapters) and synthesized by Microsynth (Balgach, Switzerland).

Each sample was digested for $2 \mathrm{~h}$ at $37^{\circ} \mathrm{C}$ in a total volume of $20 \mu \mathrm{L}$ containing $10 \mu \mathrm{L}$ DNA

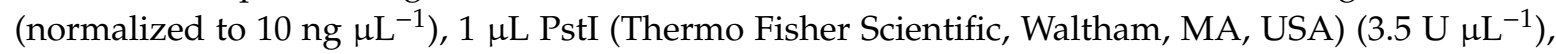
$2.5 \mu \mathrm{L}$ barcoded adaptors $\left(0.1 \mathrm{ng} \mu \mathrm{L}^{-1}\right), 2.5 \mu \mathrm{L}$ common adaptors $\left(0.1 \mathrm{ng} \mu \mathrm{L}^{-1}\right), 2 \mu \mathrm{L}$ O-buffer $(10 \times)$ and $2 \mu \mathrm{L} \mathrm{H} \mathrm{H}_{2} \mathrm{O}$. Samples were ligated with T4 DNA ligase in T4 DNA ligase buffer (10×) according to the manufacturer's instructions (Qiagen, Hilden, Germany). Equimolar amounts of each sample were pooled and then purified (Qiagen MinElute PCR Purification Kit; Qiagen, Hilden, Germany). Total volumes of $50 \mu \mathrm{L}$ ( $5 \mu \mathrm{L}$ DNA library, $0.25 \mu \mathrm{L}$ DreamTaq DNA Polymerase $\left(5 \mathrm{U}^{-1}\right), 5 \mu \mathrm{L}$ DreamTaq (10×) Buffer (Thermo Fisher Scientific, Waltham, MA, USA), $5 \mu \mathrm{L}$ dNTPs $(2 \mathrm{mM}), 1 \mu \mathrm{L}$ primers $(10 \mathrm{mM})$, and $33.75 \mu \mathrm{L} \mathrm{H}_{2} \mathrm{O}$ ) were used for the amplification of fragments. Conditions for the thermocycler were $72{ }^{\circ} \mathrm{C}$ for $5 \mathrm{~min}, 95^{\circ} \mathrm{C}$ for $30 \mathrm{~s}, 21$ cycles of $95{ }^{\circ} \mathrm{C}$ for $10 \mathrm{~s}, 65^{\circ} \mathrm{C}$ for $30 \mathrm{~s}, 72{ }^{\circ} \mathrm{C}$ for $30 \mathrm{~s}$, and $72^{\circ} \mathrm{C}$ for $5 \mathrm{~min}$ (GeneAMP PCR System 9700; Thermo Fisher Scientific, Waltham, MA, USA). The quality and length of fragments were determined on a 2200 TapeStation (Agilent Technologies, Santa Clara, CA, USA).

One 192-plex and one 60-plex GBS library of the 240 individuals were sequenced using $126 \mathrm{bp}$ single-end reads on two lanes of an Illumina HiSeq2500 platform at the Functional Genomics Center Zurich, Switzerland. Reads were demultiplexed using sabre (https://github.com/najoshi/sabre), without mismatches allowed. The reads were then aligned to the draft genome of perennial ryegrass [27] using Bowtie2 (v2.3.2) [28]. The resulting alignment files were sorted and compressed before variant calling using SAMtools (v.0.1.19) with the number of high-quality bases for each observed allele (DPR) reported [29].

\subsection{Principal Component Analysis, Hierarchical Clustering, and Permutations}

The data generated by GBS was analyzed with an in-house R-script. In brief, after filtering, samples with $>10,000$ single nucleotide polymorphisms (SNPs) were retained using the ' $v c f R$ ' package [30]. To account for differing ploidy levels, polymorphic loci were converted to allele frequencies $(0-1)$. To compare between genotypes, complete pairwise correlations were used to account for missing values, which are common for GBS. Comparisons were measured using the Pearson correlation coefficient ('cor' function). The resulting correlation matrix was then converted to a Euclidean-based distance matrix ('dist' function) and subject to agglomerative hierarchical clustering ('hclust' function) using the 'ward.D' method. Hierarchical clustering was displayed using the 'factoextra R package' ('fviz_dend' 
function). The distance matrix was subject to principal component analysis (PCA, 'prcomp' function) and permutation analysis. For each permutation, 5-55 individuals were selected randomly, then the correlation between the SNP frequencies within each subset was compared to the whole population, as described above, using the 'cor' function. Each permutation step was subject to 1000 iterations. Differences within and between populations were tested by ANOVA ('aov' function), followed by a post hoc test ('TukeyHSD' function; 0.95 confidence interval).

\subsection{Yield Trials}

The 26 tetraploid hybrid populations were tested in five independent field trials sown and harvested in the years 2014-2016, 2015-2017, 2016-2017, containing two, four, and four tetraploid synthetics as standards, respectively. The varieties of standards were released in recent years, which were continuously exchanged in sets of two. Two trials had limitations in space and duration, which resulted in a reduced number of available plots and the use of two standards only for the two harvesting years. Each trial was organized as a fully randomized block design, with each entry in triplicate. The individual trials contained a unique set of F1-hybrids derived from crosses of the previous year.

Plot size was $7.5 \mathrm{~m} \times 1.5 \mathrm{~m}$, located at GPS (decimal degrees) 53.977897, 11.468867 - 53.977462, 11.469598; 53.983284, 11.471897-53.982948, 11.472652; 53.982891, 11.471766-53.982602, 11.472524; 53.982425, 11.471629-53.982131, 11.472396; 53.982105, 11.471535-53.981741, 11.472267. Total harvest was calculated as the sum of total dry matter yield $\left(\mathrm{dt} \mathrm{ha}^{-1}\right)$ over all trial years and all individual cuttings per year.

Average population performance, measured as yield $(Y)$, was estimated using a linear model with population $(P)$ and environment (trial and year) $(E)$ as predictors with an intercept $(i)$, as shown below. Each location per year was considered independent.

$$
Y=i+P+E
$$

The prediction was used to assess the performance of hybrid populations and synthetic varieties across the trial sites and years. As a null hypothesis, no difference between hybrids and synthetic varieties was assumed. The null hypothesis was tested by ANOVA.

\section{Results}

\subsection{Genetic Structure of Selected Parental Populations from a CMS-Based Hybrid Breeding Program}

Out of the 240 genotyped individuals, 215 met the filtering criteria of at least 10,000 SNP calls across all polymorphic sites. The hierarchical clustering of these 215 individuals revealed five subgroups (Figure 1a). Within those subgroups, the 55 diploid pollinators, the 51 diploid CMS plants, and the 40 tetraploid pollinators formed separate clusters. However, the tetraploid CMS individuals were split between two distinct clusters with 39 and 16 individuals, respectively. Fourteen individuals did not cluster into their respective groups: one diploid CMS plant clustered with the tetraploid pollinators, one diploid pollinator clustered with the tetraploid CMS plants, nine tetraploid pollinators clustered with the tetraploid CMS plants, and three tetraploid CMS plants clustered with the tetraploid pollinators.

The SNP-based distance matrix was used for PCA, with the first three components explaining 30.96, 50.15, and $63.69 \%$ cumulative variance, respectively (Figure 1b, Figure S1). Individuals of the diploid CMS (yellow), diploid pollinator (grey), and tetraploid pollinator (blue) populations clustered in distinct groups. Similar to hierarchical clustering, individuals of the tetraploid CMS population (green) formed a sub-population shared with the tetraploid pollinator. 


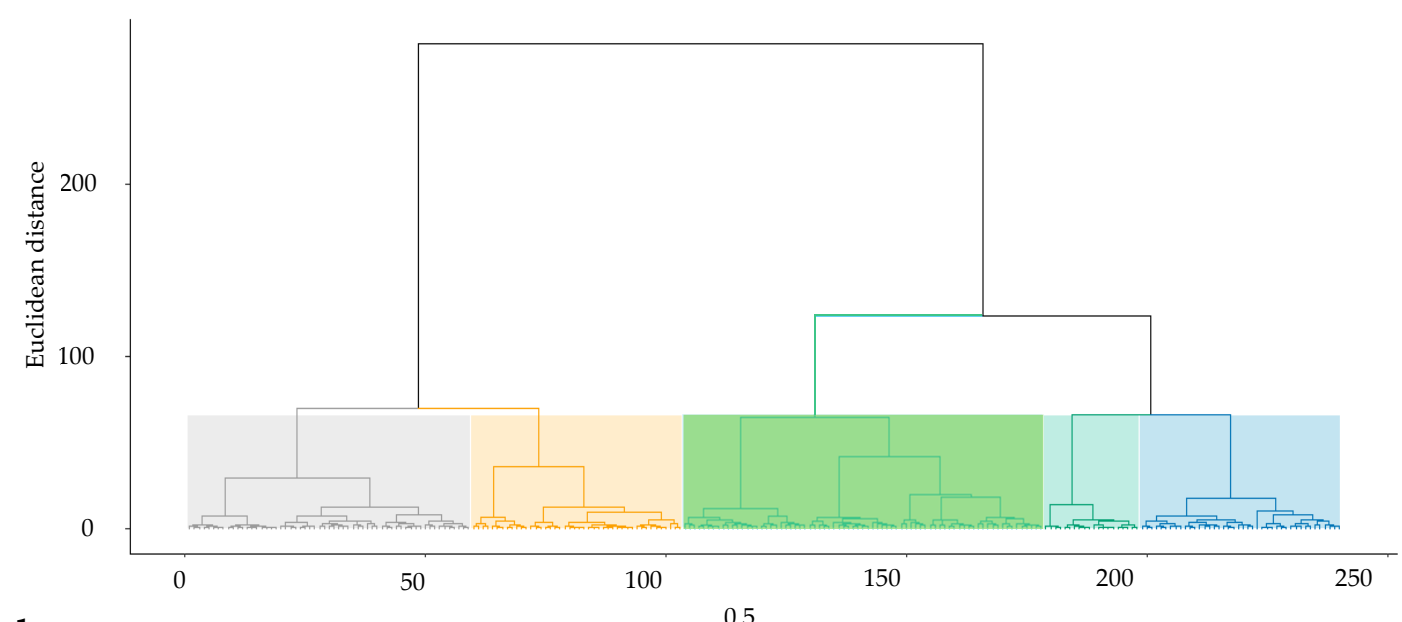

b

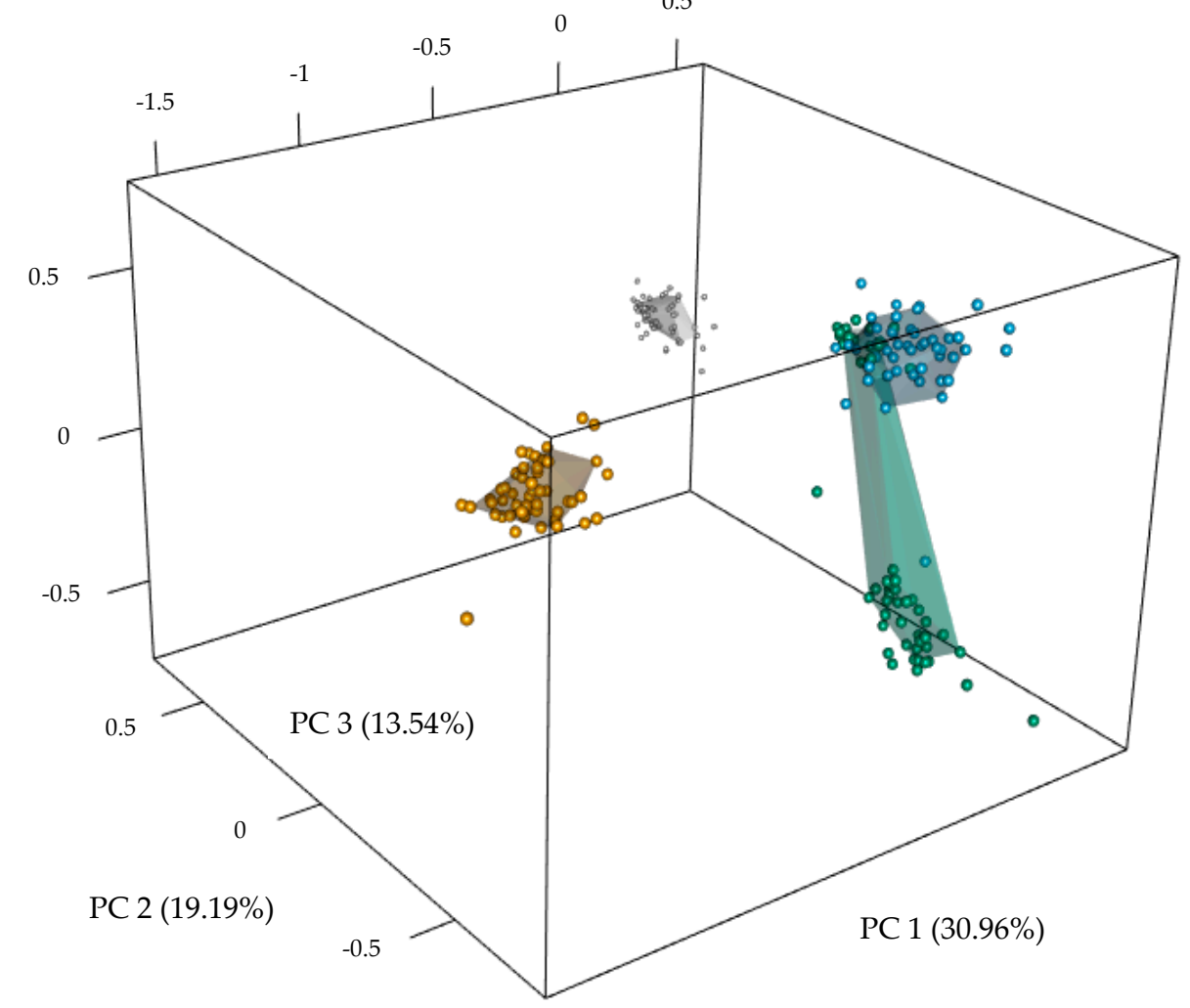

Figure 1. Genetic structure of diploid and tetraploid parental populations from cytoplasmic male sterility (CMS)-based hybrid breeding program of perennial ryegrass (Lolium perenne L.) (a) Hierarchical clustering of all individuals, showing five groups, illustrated by the sample number ( $x$-axis) and the similarity as Euclidean distance (y-axis). (b) Principal component (PC) analysis of diploid CMS (yellow), diploid pollinator (grey), tetraploid CMS (green), and tetraploid pollinator populations (blue). Shaded areas enclosed $50 \%$ of the respective colored samples. The three components-PC 1, PC 2, and PC 3-explained 30.96, 19.19, and 13.54\% of the total genotypic variance, respectively. 


\subsection{Sample Numbers Required to Represent the Allelic Composition of Breeding Populations}

In order to determine the number of individuals required to represent the allelic composition of the populations, 5-55 individuals of the diploid and tetraploid CMS and pollinator populations were randomly selected for permutation tests (Figure 2). With increasing sample numbers, all populations showed a significant increase in the saturation of the proportion of allelic frequencies. Only the increase from 50 to 55 samples of the diploid CMS population was not significantly different $(p=0.2585)$. Diploid and tetraploid CMS populations (Figure 2a) showed a significant difference $(p=0.0744)$ in the proportion of allelic frequencies for up to 45 samples. Within the pollinator populations (Figure $2 b$ ), diploids and tetraploids were similar using 20 samples and more $(p=0.9435)$. In addition, the variance of each permutation subset decreased with an increasing sample number.

a

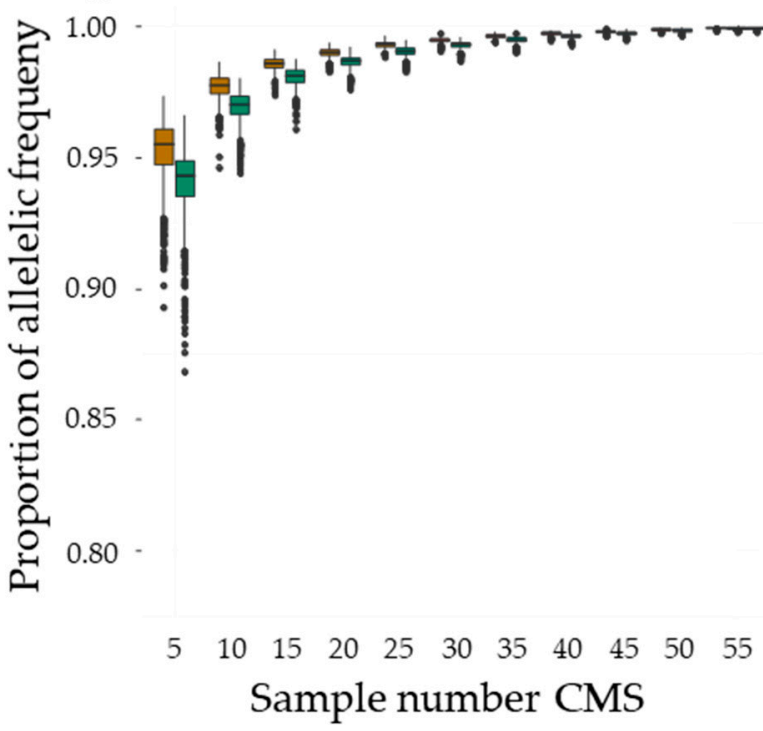

$b$

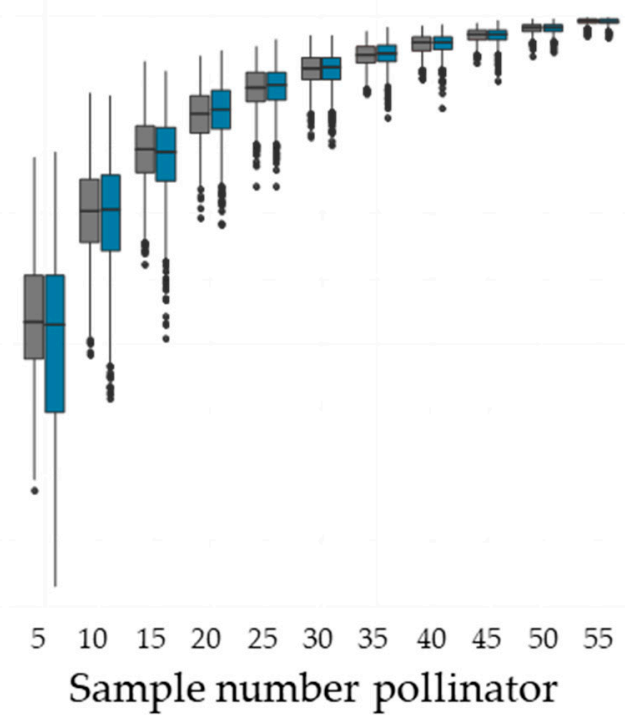

Figure 2. The proportion of allelic frequencies of diploid (yellow) and tetraploid (green) CMS populations (a) and diploid (grey) and tetraploid (blue) pollinator populations (b). The $x$-axis indicates the sample numbers used for the permutation tests, and the $y$-axis the proportion of allelic frequency.

\subsection{Performance of Tetraploid Perennial Ryegrass Hybrids and Synthetic Varieties}

In the linear model used to describe the yield performance, the estimated dry matter yield $\left(\mathrm{dt} \mathrm{ha}^{-1}\right)$ was significantly $(p<0.001)$ correlated to the observed dry matter yield $\left(R^{2}=0.96\right)$ (Figure $\left.3 \mathrm{a}\right)$. Based on the estimated yield, ANOVA revealed significant differences between hybrids and synthetics $(p=0.0155)$. The average dry matter yield performance was $429.5 \mathrm{dt} \mathrm{ha}^{-1}$ and $411.7 \mathrm{dt} \mathrm{ha}^{-1}$ for hybrids and synthetics, respectively (Figure $3 \mathrm{~b}$ ). This was equivalent to a $4.15 \%$ increased average performance of hybrids. 

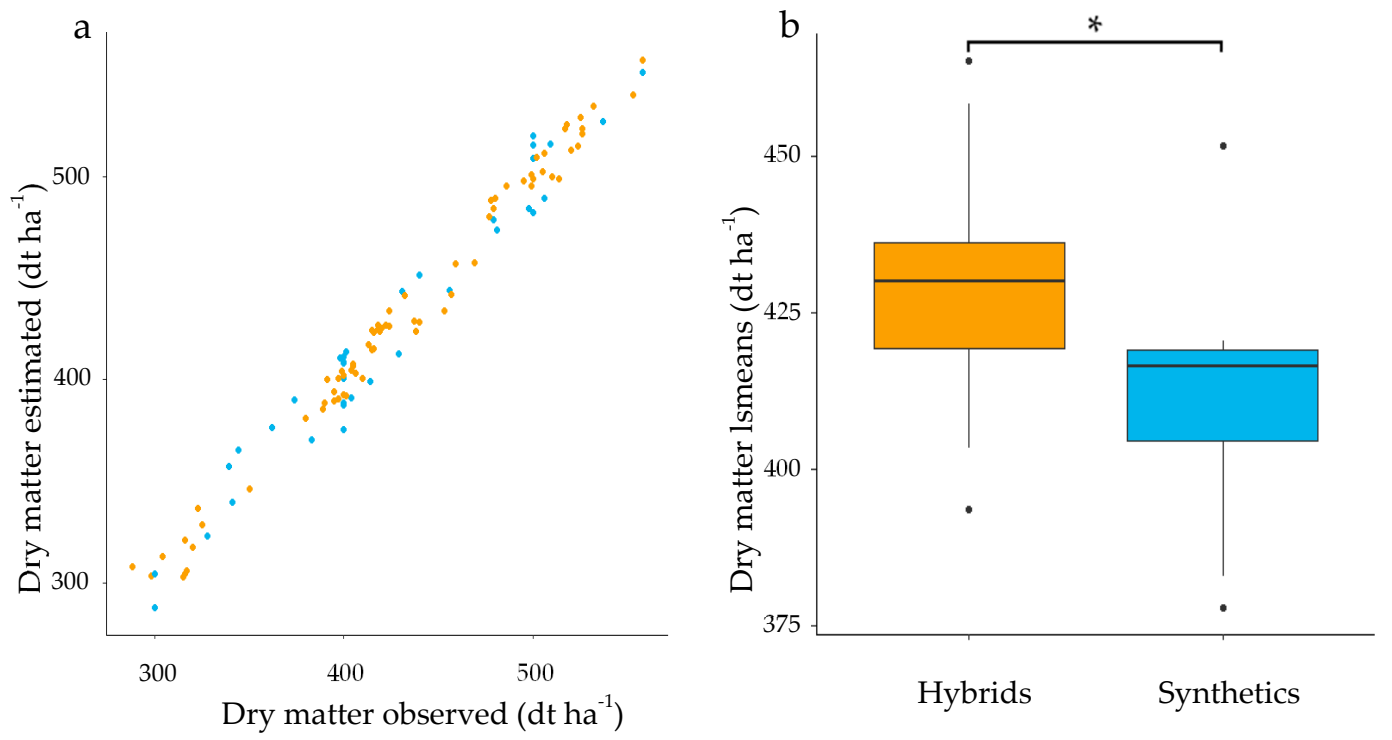

Figure 3. Results of the yield trials to test the performance of tetraploid hybrids and synthetics. (a) Estimated ( $y$-axis) versus observed ( $x$-axis) dry matter yield $\left(\mathrm{dt} \mathrm{ha}^{-1}\right)$ of perennial ryegrass hybrids (orange) and synthetics (blue); (b) Boxplot of the estimated dry matter yield ( $\mathrm{dt} \mathrm{ha}^{-1}$ ) of perennial ryegrass hybrids and synthetics. The asterisk indicates a statistical difference $(p=0.05)$.

\section{Discussion}

\subsection{Improved Yield Performance through Hybrid Breeding}

The tetraploid perennial ryegrass hybrids described here outperformed tetraploid synthetic varieties by $4.15 \%$ on average for total dry matter yield. Considering the annual genetic gain for biomass yield in perennial ryegrass, this yield increase equates to seven to ten years of breeding progress $[12,31]$. However, only the hybrid performance was assessed but not heterosis, as knowledge of parental performance is absent. In addition, the hybrids were generated by crossing CMS populations with highly heterozygous pollinator populations. This is in contrast to most hybrid breeding strategies, where parental inbred lines are crossed to maximize heterozygosity and heterosis in the F1 hybrid.

The efficient development of inbred lines in perennial ryegrass is hampered by an effective SI mechanism, preventing seed production from selfings. Additionally, SI and subsequent outcrossing have allowed for the accumulation of deleterious alleles in the evolutionary history of Lolium spp., leading to severe inbreeding depression in selfed breeding material. Overcoming SI and hence the prevention of inbreeding would not only enable to increase the degree of homozygosity in parental plant materials but also purge breeding germplasm from deleterious alleles with recurrent cycles of self-pollination. This could lead to the development of superior inbred lines to be used as parents in hybrid breeding schemes, untapping the potential to further increase biomass yields in F1 hybrids [5,32].

\subsection{Identification of Heterotic Groups is Key for Successful and Efficient Hybrid Breeding in Perennial Ryegrass}

Genotyping-by-sequencing allowed us to identify heterotic groups within the diploid and tetraploid parental breeding populations. This is a major improvement compared to unstructured germplasm, as identification of heterotic groups is not only pivotal to fully exploit the potential of hybrid breeding but also to improve the efficiency of CMS-based hybrid breeding strategies $[17,25,33]$. Using CMS, it is possible to test the general combining abilities of selected populations within the breeding material [34]. In practice, however, only a fraction of the possible parental combinations can be evaluated in each cycle [17]. The factors constraining testcross combinations include technical (seed production, space) and biological (non-overlapping flowering times) limitations [17,35]. Thus, the availability of marker data within and between heterotic groups will be helpful to allocate the 
often limited resources of a breeding program to the most promising parental combinations for testcross evaluation.

Previous studies have presented methods where genotyping has been performed on pooled samples to infer population allele frequencies $[26,36,37]$. These approaches have been successful in distinguishing varieties, aiming to support breeders' rights and to improve variety registration. However, they have failed to identify the detailed structure within population-based germplasm and to support the development of heterotic groups [26,38]. In contrast, the individual-based genotyping strategy described here not only allows precise germplasm fingerprinting, but it also provides a solid basis to develop and further diverge heterotic groups using marker data of individual plants. With the highly multiplexed GBS protocol applied here, genotyping of up to 1536 individuals is possible with a single run on an Illumina HiSeq2500 instrument. Additional flexibility and throughput of DNA sequencing can be realized using the novel Illumina NovaSeq 6000 or similar systems, further consolidating GBS as a time- and cost-effective tool to genotype single individuals. By doing so, breeders can assign individuals of elite populations into distinct heterotic groups. For this purpose, genotyping 20-45 individuals is sufficient to capture, on average, $99 \%$ of the genetic variation and to detect concealed heterotic groups within a population. As the development of any heterotic group requires a minimum of 8-16 plants, genotyping of at least 60 individuals is recommended to form new breeding populations. Diversification and development of heterotic groups could potentially lead to similar success in hybrid breeding, as seen in maize, canola, rye, and other crops [19-21,26].

\subsection{Evidence for Sub-Populations within Heterotic Groups as Revealed by Genotyping-by-Sequencing}

Interestingly, the proportion of allelic frequencies within the diploid and tetraploid pollinator populations appears to be higher when compared to their respective CMS populations. There are a number of potential reasons for this difference in the genetic make-up of the pollinator and the CMS populations. For example, the CMS trait has been introgressed from a single founder plant. This could have influenced the selection of alleles that are necessary to maintain the male sterility within CMS populations. Additionally, CMS populations have been subject to seed propagation by backcrossing to their maintainer populations. This could have driven the emergence of sub-populations and amplify their diversity based on the allelic constitution at SI loci. Finally, SI could also have played a role in maintaining high diversity by frequency-dependent selection in pollinator populations: the phenotypic selection of plants with high seed yield may have favored pollen contaminations with novel SI alleles [5].

In the context of hybrid breeding, the overlap between heterotic groups increases the degree of homozygosity in the resulting hybrids and consequently impede hybrid performance. Our observations in the tetraploid CMS germplasm described here illustrate the need to genotype single plants to develop and maintain breeding populations. An exhaustive analysis of populations from the CMS hybrid breeding program beyond the four characterized in our study could identify more populations harboring multiple groups, thus providing a method to identify complementary germplasm and to develop heterotic groups by directed crosses. Both are key to successful hybrid breeding for perennial ryegrass [20].

\section{Conclusions}

Averaged across multiple evaluation sites and years, the biomass yield performance of CMS-based population hybrids in tetraploid perennial ryegrass was significantly higher compared to currently used synthetic cultivars. Detailed analysis of the genetic structure of parental populations using molecular tools will help to realize the untapped potential to further increase biomass yields. Genotyping-by-sequencing is a useful tool to characterize diploid and tetraploid breeding populations of perennial ryegrass and possibly other crops in order to identify population structures. Through the continuous development of populations towards distinct heterotic groups, the potential of heterosis can be exploited more efficiently. 
Supplementary Materials: The following are available online at http://www.mdpi.com/2073-4395/10/9/1410/s1, Figure S1: Cumulative proportion of explained variance of the principal component analysis (PCA) used to describe the genetic structure of selected diploid and tetraploid parental populations from a cytoplasmic male sterility (CMS)-based hybrid breeding program of perennial ryegrass (Lolium perenne L.).

Author Contributions: Conceptualization, M.V., T.S., W.L., and B.S.; Formal analysis, M.V. and S.Y.; Investigation, M.V.; Project administration, W.L. and B.S.; Resources, W.L., M.K., and B.S.; Supervision, W.L., M.K., and B.S.; Visualization, M.V.; Writing—original draft, M.V., S.Y., and B.S.; Writing—review and editing, S.Y., M.K., and B.S. All authors have read and agreed to the published version of the manuscript.

Funding: This program has received funding from the European Union's Horizon 2020 research and innovation program under the Marie Skłodowska-Curie grant agreement No 722338-PlantHUB.

Acknowledgments: We thank the Zurich-Basel Plant Science Center for professional management of the PlantHUB program and Verena Knorst-Rashid for excellent caretaking of the plant material. Library preparation and DNA sequencing were kindly supported by the Genetic Diversity Center of ETH Zurich and the Functional Genomics Center Zurich, Switzerland.

Conflicts of Interest: The authors declare no conflict of interest.

\section{References}

1. Wilkins, P.W.; Humphreys, M.O. Progress in breeding perennial forage grasses for temperate agriculture. J. Agric. Sci. 2003, 140, 129-150. [CrossRef]

2. Bothe, A.; Westermeier, P.; Wosnitza, A.; Willner, E.; Schum, A.; Dehmer, K.J.; Hartmann, S. Drought tolerance in perennial ryegrass (Lolium perenne L.) as assessed by two contrasting phenotyping systems. J. Agron. Crop. Sci 2018, 204, 375-389. [CrossRef]

3. Loka, D.; Harper, J.; Humphreys, M.; Gasior, D.; Wootton-Beard, P.; Gwynn-Jones, D.; Scullion, J.; Doonan, J.; Kingston-Smith, A.; Dodd, R.; et al. Impacts of abiotic stresses on the physiology and metabolism of cool-season grasses: A review. Food Energy Secur. 2019, 8, e00152. [CrossRef]

4. Cyriac, D.; Hofmann, R.W.; Stewart, A.; Sathish, P.; Winefield, C.S.; Moot, D.J. Intraspecific differences in long-term drought tolerance in perennial ryegrass. PLoS ONE 2018, 13, e0194977. [CrossRef] [PubMed]

5. Do Canto, J.; Studer, B.; Lubberstedt, T. Overcoming self-incompatibility in grasses: A pathway to hybrid breeding. Theor. Appl. Genet. 2016, 129, 1815-1829. [CrossRef]

6. Hayward, M.D.; Abdullah, I.B. Selection and stability of synthetic varieties of Lolium perenne. Theor. Appl. Genet. 1985, 70, 48-51. [CrossRef]

7. Casler, M.D.; Brummer, E.C. Theoretical Expected Genetic Gains for Among-and-Within-Family Selection Methods in Perennial Forage Crops. Crop. Sci. 2008, 48, 890-902. [CrossRef]

8. Aguirre, A.A.; Studer, B.; Frei, U.; Lubberstedt, T. Prospects for Hybrid breeding in Bioenergy Grasses. Bioenergy Res. 2012, 5, 10-19. [CrossRef]

9. Carew, R.; Florkowski, W.J.; Meng, T. Intellectual property rights and plant variety protection of horticultural crops: Evidence from Canada. Can. J. Plant. Sci. 2017, 97, 737-754. [CrossRef]

10. Halpert, M.-T.; Chappell, M.J. Prima facie reasons to question enclosed intellectual property regimes and favor open-source regimes for germplasm. F1000Research 2017, 6, 284. [CrossRef]

11. Blackmore, T.; Thorogood, D.; Skøt, L.; McMahon, R.; Powell, W.; Hegarty, M. Germplasm dynamics: The role of ecotypic diversity in shaping the patterns of genetic variation in Lolium perenne. Sci. Rep. 2016, 6, 22603. [PubMed]

12. Pembleton, L.W.; Shinozuka, H.; Wang, J.; Spangenberg, G.C.; Forster, J.W.; Cogan, N.O.I. Design of an F1 hybrid breeding strategy for ryegrasses based on selection of self-incompatibility locus-specific alleles. Front. Plant Sci. 2015, 6, 764. [PubMed]

13. Chen, E.; Huang, X.; Tian, Z.; Wing, R.A.; Han, B. The Genomics of Oryza Species Provides Insights into Rice Domestication and Heterosis. Annu. Rev. Plant Biol. 2019, 70, 639-665.

14. Kempe, K.; Gils, M. Pollination control technologies for hybrid breeding. Mol. Breed. 2011, 27, 417-437.

15. Islam, M.S.; Studer, B.; Møller, I.M.; Asp, T. Genetics and biology of cytoplasmic male sterility and its applications in forage and turf grass breeding. Plant Breed. 2014, 133, $299-312$. 
16. Bolaric, S.; Barth, S.; Melchinger, A.E.; Posselt, U.K. Molecular characterization of genetic diversity in European germplasm of perennial ryegrass. Euphytica 2005, 146, 39-44.

17. Zhao, Y.; Li, Z.; Liu, G.; Jiang, Y.; Maurer, H.P.; Würschum, T.; Mock, H.-P.; Matros, A.; Ebmeyer, E.; Schachschneider, R.; et al. Genome-based establishment of a high-yielding heterotic pattern for hybrid wheat breeding. Proc. Natl. Acad. Sci. USA 2015, 112, 15624-15629.

18. Posselt, U.K. Hybrid production in Lolium perenne based on incompatibility. Euphytica 1993, 71, $29-33$.

19. Duvick, D.N. Biotechnology in the 1930s: The development of hybrid maize. Nat. Rev. Genet. 2001, 2, 69-74.

20. Becker, H.C.; Löptien, H.; Röbbelen, G. Breeding: An overview. In Developments in Plant Genetics and Breeding; Gómez-Campo, C., Ed.; Elsevier: Amsterdam, The Netherlands, 1999; Volume 4, pp. 413-460.

21. Miedaner, T.; Korzun, V.; Bauer, E. Chapter 15-Genomics-Based Hybrid Rye Breeding. In Applications of Genetic and Genomic Research in Cereals; Miedaner, T., Korzun, V., Eds.; Woodhead Publishing: Sawston, UK, 2019; pp. 329-348, ISBN 9780081021637.

22. Schlegel, R. Hybrid breeding boosted molecular genetics in rye. Russ. J. Genet. Appl. Res. 2016, 6, 569-583.

23. Guan, X.; Yuyama, N.; Stewart, A.; Ding, C.; Xu, N.; Kiyoshi, T.; Cai, H. Genetic Diversity and Structure of Lolium Species Surveyed on Nuclear Simple Sequence Repeat and Cytoplasmic Markers. Front. Plant Sci. 2017, 8, 584. [CrossRef] [PubMed]

24. Miedaner, T.; Glass, C.; Dreyer, F.; Wortmann, P.W.H.; Geiger, H.H. Mapping of genes for male-fertility restoration in 'Pampa' CMS winter rye (Secale cereale L.). Theor. Appl. Genet. 2000, 101, 1226-1233. [CrossRef]

25. Laidig, F.; Piepho, H.-P.; Rentel, D.; Drobek, T.; Meyer, U.; Huesken, A. Breeding progress, variation, and correlation of grain and quality traits in winter rye hybrid and population varieties and national on-farm progress in Germany over 26 years. Theor. Appl. Genet. 2017, 130, 981-998. [CrossRef] [PubMed]

26. Liu, S.; Feuerstein, U.; Luesink, W.; Schulze, S.; Asp, T.; Studer, B.; Becker, H.C.; Dehmer, K.J. DArT, SNP, and SSR analyses of genetic diversity in Lolium perenne L. using bulk sampling. BMC Genet. 2018, 19, 10. [CrossRef]

27. Byrne, S.L.; Nagy, I.; Pfeifer, M.; Armstead, I.; Swain, S.; Studer, B.; Mayer, K.; Campbell, J.D.; Czaban, A.; Hentrup, S.; et al. A synteny-based draft genome sequence of the forage grass Lolium perenne. Plant J. 2015, 84, 816-826. [CrossRef]

28. Langmead, B.; Trapnell, C.; Pop, M.; Salzberg, S.L. Ultrafast and memory-efficient alignment of short DNA sequences to the human genome. Genome Biol. 2009, 10, R25. [CrossRef]

29. Li, H. A statistical framework for SNP calling, mutation discovery, association mapping and population genetical parameter estimation from sequencing data. Bioinformatics 2011, 27, 2987-2993. [CrossRef]

30. Knaus, B.J.; Grünwald, N.J. vcfr: A package to manipulate and visualize variant call format data in R. Mol. Ecol. Resour. 2017, 17, 44-53. [CrossRef]

31. Humphreys, M.O. The contribution of conventional plant breeding to forage crop improvement. In Proceedings of the 18th International Grassland Congress, Winnipeg and Saskatoon, SK, Canada, 8-17 June 1997.

32. Conaghan, P.; Casler, M.D. A theoretical and practical analysis of the optimum breeding system for perennial ryegrass. Ir. J. Agric. Food Res. 2011, 50, 47-63.

33. Posselt, U.K. Identification of Heterotic Patterns in Perennial Ryegrass. In Sustainable Use of Genetic Diversity in Forage and Turf Breeding; Springer: Dordrecht, The Netherlands, 2010; pp. 569-572.

34. Yingheng, W.; Qiuhua, C.; Hongguang, X.; Fangxi, W.; Ling, L.; Wei, H.; Liping, C.; Hua'an, X.; Jianfu, Z. Determination of Heterotic Groups and Heterosis Analysis of Yield Performance in indica Rice. Rice Sci. 2018, 25, 261-269. [CrossRef]

35. Dey, S.S.; Sharma, S.R.; Bhatia, R.; Parkash, C.; Barwal, R.N. Superior CMS (Ogura) lines with better combining ability improve yield and maturity in cauliflower (Brassica oleracea var. botrytis). Euphytica 2011, 182, 187. [CrossRef]

36. Byrne, S.; Czaban, A.; Studer, B.; Panitz, F.; Bendixen, C.; Asp, T. Genome wide allele frequency fingerprints (GWAFFs) of populations via genotyping by sequencing. PLoS ONE 2013, 8, e57438. [CrossRef] 
37. Hale, M.L.; Burg, T.M.; Steeves, T.E. Sampling for microsatellite-based population genetic studies: 25 to 30 individuals per population is enough to accurately estimate allele frequencies. PLoS ONE 2012, 7, e45170. [CrossRef]

38. Pembleton, L.W.; Drayton, M.C.; Bain, M.; Baillie, R.C.; Inch, C.; Spangenberg, G.C.; Wang, J.; Forster, J.W.; Cogan, N.O.I. Targeted genotyping-by-sequencing permits cost-effective identification and discrimination of pasture grass species and cultivars. Theor. Appl. Genet. 2016, 129, 991-1005. [CrossRef] 